David Mondada - Fredrik T. Bosman •

Charlotte Fontolliet - Walter K. F. Seelentag

\title{
Elevated hepatocyte paraffin 1 and neprilysin expression in hepatocellular carcinoma are correlated with longer survival
}

Received: 14 April 2005 / Accepted: 25 August 2005 / Published online: 12 October 2005

(C) Springer-Verlag 2005

\begin{abstract}
Hepatocyte paraffin 1 (Hep Par 1) and neprilysin (CD10) are well-known markers of hepatocellular carcinoma (HCC). To assess their potential prognostic role, we conducted a retrospective analysis of 97 formalin-fixed and paraffin-embedded HCC from patients treated by surgery with curative intent, using standard immunohistochemical procedures and semiquantitative analysis. Strong Hep Par 1 expression and canalicular CD10 staining pattern were significantly correlated with smaller tumor size ( $p=0.007$ and 0.04 , respectively). On univariate analysis, longer overall survival was observed in patients with strong Hep Par 1 expression $(p=0.0005)$ and in patients with a CD10can staining pattern $(p=0.02)$. On multivariate analysis, the combined immunohistochemical score (CIS) obtained by addition of Hep Par 1 and CD10can scores and subtraction of cytoplasmic CD10 score was retained as the single most important prognostic factor $(p=0.001)$. Patients with a CIS $<4$ had a 3.5 -fold increased risk of death, as compared to those with a CIS $\geq 4$. In conclusion, strong Hep Par 1 expression, presence of CD10can labeling, and absence of CD10cyt staining are favorable prognostic factors in $\mathrm{HCC}$, which can be easily combined into a single immunohistochemical score for routine clinical use.
\end{abstract}

Keywords Carcinoma $\cdot$ Hepatocellular . Immunohistochemistry $\cdot$ Antibodies $\cdot$ Monoclonal Prognosis

D. Mondada · F. T. Bosman · C. Fontolliet .

W. K. F. Seelentag $(\bowtie)$

Institute of Pathology, University of Lausanne,

Bugnon 25,

1011 Lausanne, Switzerland

e-mail: Walter.Seelentag@hospvd.ch

Tel.: +41-21-3147208

Fax: +41-21-3147115

\section{Introduction}

Prognosis of hepatocellular carcinoma (HCC) after hepatic resection with curative intent remains very poor despite improved surgical techniques $[17,23,26]$. In addition, in low endemic areas like Switzerland, HCC is often discovered in elderly patients with a long-standing alcoholic cirrhosis and at high operative risk. Therefore, adequate prognostic stratification of the patients to guide the clinician in treatment choice is essential. The most important histopathologic prognostic factors are tumor size, vascular invasion, tumor multifocality, and tumor grade $[1,24,29$, 30]. Combined scores based on histological features or image analysis have also been proposed [23]. In addition, the prognostic significance of a variety of gene products related to the cell cycle or to cell adhesion has been described in the literature, such as the tumor-suppressor gene p53, the cell cycle inhibitors p21, p27, and cyclin D, and the cell adhesion molecule beta-catenin $[18,20,31]$. However, none of these markers have found their way into routine clinical use.

Hepatocyte paraffin 1 (Hep Par 1) [2, 21, 27, 38] and neprilysin (CD10 or CALLA) [5, 40] are well-established markers of HCC, and their use as diagnostic aid is well documented in the literature. Hep Par 1 has been obtained by immunizing mice with a crude antigen extracted from human liver tissue [38]. It reacts with a hepatocyte-specific epitope of the mitochondrial membrane [21, 22, 38], resulting in a granular cytoplasmic staining pattern. This marker has been shown to be highly specific for normal and neoplastic liver tissue. CD10 is a zinc-dependant cell membrane metallopeptidase, which participates in the postsecretory processing of neuropeptidases and peptide hormones [37]. In the past, it has been widely used as a marker of lymphoblasts in acute lymphoblastic leukemia [4]. More recently, it has also been shown that CD10 is expressed on the canalicular domain of the cell membrane in normal and neoplastic liver tissue [7, 11, 12].

Specificity of these two markers has been well established in the literature, allowing the distinction of $\mathrm{HCC}$ 
from cholangiocarcinomas and carcinoma metastases to the liver [25, 27, 28]. Surprisingly, to our knowledge, despite several studies on the diagnostic value of these markers and their association with tumor grade and growth pattern, the possible association of their expression with survival has never been examined in detail. In reviewing our pilot series of HCC, the extent of Hep Par 1 and canalicular CD10 (CD10can) expression seemed to be related to the degree of differentiation. In contrast, cytoplasmic CD10 (CD10cyt) expression seemed to be restricted to cases with low Hep Par 1 and CD10can expression. We therefore decided to systematically evaluate the prognostic value of these three parameters. In the current retrospective study, we analyze the immunohistochemical expression of Hep Par 1 and CD10 in formalin-fixed and paraffin-embedded HCC tissue from patients who underwent either partial hepatectomy with curative intent or total hepatectomy followed by liver transplantation to assess their prognostic value.

\section{Patients and methods}

\section{Patients and tissue samples}

All patients $(n=122)$ who underwent either partial hepatectomy with curative intent or total hepatectomy with liver transplantation because of primary HCC and who were diagnosed at the Institute of Pathology in Lausanne, Switzerland, between January 1992 and May 2001 were selected for this retrospective study. Excluded were patients with mixed hepato-choloangiocarcinoma $(n=8)$, fibrolamellar carcinoma $(n=1)$, inadequate follow-up $(n=11)$, missing material for immunohistochemistry $(n=2)$, and patients who died within 30 days after diagnosis $(n=3)$. The ethics committee of our institution approved the study protocol.

The remaining 97 patients consisted of typical Western European patients whose clinical characteristics were similar to those of several other published series $[9,26,32]$. Treatment was partial liver resection in 72 patients $(74 \%)$ and total hepatectomy followed by liver transplantation in 25 patients $(26 \%)$. Most patients $(78 \%)$ were men. The median age was 63 years (range 14 to 89 years) and was similar for male and female patients. Cirrhosis was found in 67 patients $(69 \%)$. Serology was positive for hepatitis B virus surface antigen in 10 patients $(10 \%)$ and hepatitis $C$ virus antigen in 18 patients (19\%). Preoperative aspartate aminotransferase, alanine aminotransferase, gamma glutamyl transferase, and alpha-fetoprotein (AFP) levels were also recorded. Overall survival time was calculated from date of diagnosis to date of death or date of last follow-up until closure of the study which was chosen at 30 August 2002. Intermediate outcomes were not considered. Median follow-up time computed according the Kaplan-Meier estimate of potential follow-up was 47 months. Overall median survival time was 28 months in the whole cohort and 23 months in the subgroup of patients treated with partial hepatectomy.

All specimens were fixed in $10 \%$ buffered formalin and embedded in paraffin according to standard histological practice. All available hematoxylin/eosin-stained sections (mean 3, range 1 to 12) were reviewed by two experienced pathologists (D.M. and C.F.), and a representative paraffin block containing both carcinoma and nontumorous tissue was selected for immunohistochemistry. Tumor stage was determined using the International Union against Cancer's tumor-nodes-metastasis (TNM) classification of malignant tumors [34]. Because of small sample size, stages pT1 and pT 2 were grouped together. Tumors were divided into grades 1, 2, 3, and 4 according to Edmondson and Steiner [13]. In the case of a heterogeneous tumor, the highest grade was retained for the database. Classification of tumor architecture into trabecular, acinar, compact, and scirrhous growth pattern was performed according to the World Health Organization's criteria [16]. Because of relatively low numbers of tumors in the various non-trabecular categories, they were grouped together for statistical analysis. Tumor size, tumor multifocality (solitary nodules/multiple nodules), and microvascular invasion (MVI) were also recorded. Hepatocellular Prognostic Index (HPI) was computed according to Lauwers et al. [23], using the following formula: HPI $=($ MVI status $\times 0.459)+($ nuclear grade $\times$ $0.287)$. Two prognostic groups were derived from this index: HPI-low (HPI $\leq 0.746)$, corresponding to patients with rather good prognosis according to Lauwers et al. [23], and HPI-high (HPI $>0.746)$, corresponding to patients with poor prognosis.

With regard to tumor stage, 33 patients (34\%) had stages I and II disease, 29 (30\%) had stage III, and 35 had (36\%) stage IV. Most HCC were less than $5 \mathrm{~cm}$ in size $(n=58$, $60 \%)$, solitary $(n=54,56 \%)$, and with a trabecular architecture $(n=75,77 \%)$. The tumor differentiation was grade 1 in $15 \mathrm{HCC}(15 \%)$, grade 2 in $47(48 \%)$, grade 3 in 27 $(28 \%)$, and grade 4 in $8(8 \%)$. MVI was present in $35 \mathrm{HCC}$ $(36 \%)$. HPI was high in 51 tumors $(53 \%)$.

\section{Immunohistochemistry}

Immunohistochemistry was performed with mouse monoclonal antibodies directed against Hep Par 1 (clone OCH1E5, DAKO) and CD10 (clone 56C6, Novocastra) using the streptavidin biotin complex (ABC) method. In brief, 3-4 $\mu$ tissue sections were mounted on aminopropylmethoxysilane-coated slides, deparaffinized in xylene, and rehydrated in a graded alcohol series. Endogenous peroxidase was blocked with $1 \% \mathrm{H}_{2} \mathrm{O}_{2}$ in methanol for $30 \mathrm{~min}$ at room temperature. Antigen retrieval was performed by boiling the sections (microwave) in $10 \mathrm{mM}$ citrate buffer $\mathrm{pH} 6.0$ for $15 \mathrm{~min}$ (Hep Par 1) or $1 \mathrm{mM}$ in EDTA pH 7.5 for $15 \mathrm{~min}$ (CD10). After conditioning with normal horse serum at a 1:30 dilution for $5 \mathrm{~min}$, the sections were incubated either with anti-Hep Par 1 at a 1:10 dilution for $1 \mathrm{~h}$ or with antiCD10 at a 1:20 dilution overnight. Detection was performed using biotinylated anti-mouse $\operatorname{IgG}$ at a 1:200 dilution and peroxidase-conjugated $\mathrm{ABC}$ complex (both from Vector Laboratories) according to the manufacturer's specifications. Between all steps, sections were washed in Tris-buffered saline. Peroxidase activity was revealed with 
3,3-diaminobenzidine-tetrahydrochloride as chromogen, and sections were counterstained with Mayer's hematoxylin.

Primary antibodies were omitted as a negative control. Nontumorous liver tissue adjacent to $\mathrm{HCC}$ and present on the same slide was used as positive control.

The immunohistochemical Hep Par 1 and CD10 staining of all slides was evaluated independently by two observers (D.M. and W.K.F.S) in a blinded manner. Divergent scorings were discussed until a consensus was reached. To assess reproducibility, a subset of slides was analyzed by a third pathologist (F.T.B.), who had not done the initial evaluation based on the information contained in this manuscript without additional training. Individual tumor cells were considered to be positive for Hep Par 1 when their cytoplasmic staining intensity was similar or stronger than that of the adjacent nontumorous hepatocytes. CD10cyt staining was considered to be positive in tumor cells when the cytoplasm showed unambigous labeling in contrast to the preexisting hepatocytes which were negative. Positive CD10can staining was defined as crisp focal labeling of the canalicular aspects of the tumor cell membrane when its intensity was similar or stronger than that of the nontumorous canaliculi.

Based on the percentage of positive cells, Hep Par 1, CD10can, and CD10cyt immunolabelings were semiquantitatively classified into four groups, based on a priori determined cutoff points. In the first group, less than $5 \%$ of the tumor cells were positive (score 0 ); in the second group, 5 to $30 \%$ of the tumor was positive (score 1); in the third group, 30 to $90 \%$ of the tumor cells were positive (score 2); and in the last group, more than $90 \%$ of the tumor was positive (score 3 ).

A combined immunohistochemical score (CIS) was computed as follows: CIS $=$ Hep Par 1 score + CD10can score - CD10cyt score.

\section{Statistical analysis}

Correlation tests between immunohistochemical and clinicopathological parameters were performed using either Kruskal-Wallis tests (for continuous or ordered variables such as age, HPI, or tumor grade) or Chi-squared tests (for categorical variables), unless there was concern regarding an inadequate number of observations, in which case, a Fisher's exact test was used [3].

Overall interobserver agreement was compared using kappa statistics. According to common usage, kappa values of $\leq 0.5$ were considered "poor", 0.51-0.6 "moderate", $0.61-0.8$ "good", and $>0.8$ "excellent".

For Fisher's exact tests and survival analysis, clinicopathological and immunohistochemical variables were dichotomized into groups of similar size as follows: age $<65 \mathrm{vs.}$ $\geq 65$ years, tumor size $\leq 5$ vs. $>5 \mathrm{~cm}$, HPI $\leq 0.746$ (low) vs. $>0.746$ (high), Hep Par $1<3$ vs. $=3$, CD 10 can $<2$ vs. $\geq 2$, CD10cyt $<1$ vs. $\geq 1$, and CIS $<4$ vs. $\geq 4$.

Survival percentages over time were calculated by the Kaplan-Meier method [19], and their corresponding standard errors were determined using the formula of Greenwood [15]. Univariate associations between the potential prognostic factors and overall survival were tested using the log-rank test.

Prognostic variables which had been at least marginally significant in univariate analysis (log-rank test $<0.1)$ were further analyzed in various Cox regression models using backward selection with $p<0.05$ as the exit criterion.

Given the relatively small number of outcome events, several distinct Cox regression models with a limited number of covariates had to be evaluated [6].

Estimated hazard ratios of death, with respect to the indicated reference group, their $95 \%$ confidence intervals (CI), and $p$ values were calculated with appropriate binary variables to identify each group of interest [10]. Values of hazard ratios greater than unity indicated increased rates of death with respect to the chosen reference category.

To evaluate the impact of type of surgery, univariate and multivariate analysis were done on the whole study population as well as on the subgroup of patients with partial liver resection.

Statistical analyses were carried out by means of the software packages Stata, S-plus 2000, and SPSS.

All probability values were evaluated by two-sided tests, and differences were considered to be significant if the $p$ value was less than 0.05 .

\section{Results}

Correlation of Hep Par 1 and CD10 immunolabeling with clinicopathological features

Hep Par 1 resulted in a diffuse labeling of the cytoplasm of nontumorous hepatocytes, which served as internal positive control, with a distinctive granular pattern. It was independent of the underlying liver pathology, and there was no staining difference between cirrhotic and non-cirrhotic liver tissue. Most HCC showed a similar cytoplasmic labeling, albeit at a highly variable intensity (Fig. 1a-c). Hep Par 1 expression, evaluated semiquantitatively as outlined in "Patients and methods", was extensive (score 3) in $54(55 \%) \mathrm{HCC}$, partial (score 2) in $31(32 \%) \mathrm{HCC}$, and focal or absent (score 1 or 0 ) in $13(13 \%)$ HCC.

CD10 labeled in nontumorous liver exclusively the luminal portion of bile canaliculi of the hepatocytes as well as the luminal surface of the bile duct epithelium. There was no staining difference between cirrhotic and noncirrhotic liver. In HCC, a similar canalicular staining pattern was seen forming sharp branching lines which were typically located between cells and not within them (Fig. 1d-f). CD10can labeling was widespread (score 3) in $20(21 \%) \mathrm{HCC}$, partial (score 2) in $38(39 \%) \mathrm{HCC}$, focal (score 1) in $11(11 \%) \mathrm{HCC}$, and completely absent in 28 (29\%) HCC. In addition, some HCC displayed a CD10cyt labeling (Fig. 1g,h), and very few tumors exhibited a diffuse cell membrane staining. This type of staining was not seen in nontumorous hepatocytes. CD10cyt staining 
Fig. 1 Representative examples of immunohistochemical staining in HCC for Hep Par $1(\mathbf{a}-\mathbf{c})$ and $\operatorname{CD} 10(\mathbf{d}-\mathbf{h})$ : individual tumor cells $(t u)$ are considered positive for Hep Par 1 when their cytoplasmic immunolabeling is similar to that of adjacent nontumorous hepatocytes $(n)$. CD10 labeling is considered to be canalicular $(\mathbf{d}-\mathbf{f})$ when there is a crisp linear staining between adjacent tumor cells (inset, d). CD10cyt staining is considered significant if individual tumor cells show an unambigous cytoplasmic staining in contrast to surrounding nontumorous liver tissue which is negative $(\mathbf{g}-\mathbf{h})$. Scoring is based on the percentage of positive tumor cells: score 3, more than $90 \%$ labeled tumor cells (a, d, and $\mathbf{g}$ ); score 2, between 30 and $90 \%$ (b, e, and $\mathbf{h}$ ); and score 1 , less than $30 \%$ positive tumor cells (c and f). Scale bars $100 \mu \mathrm{m}$
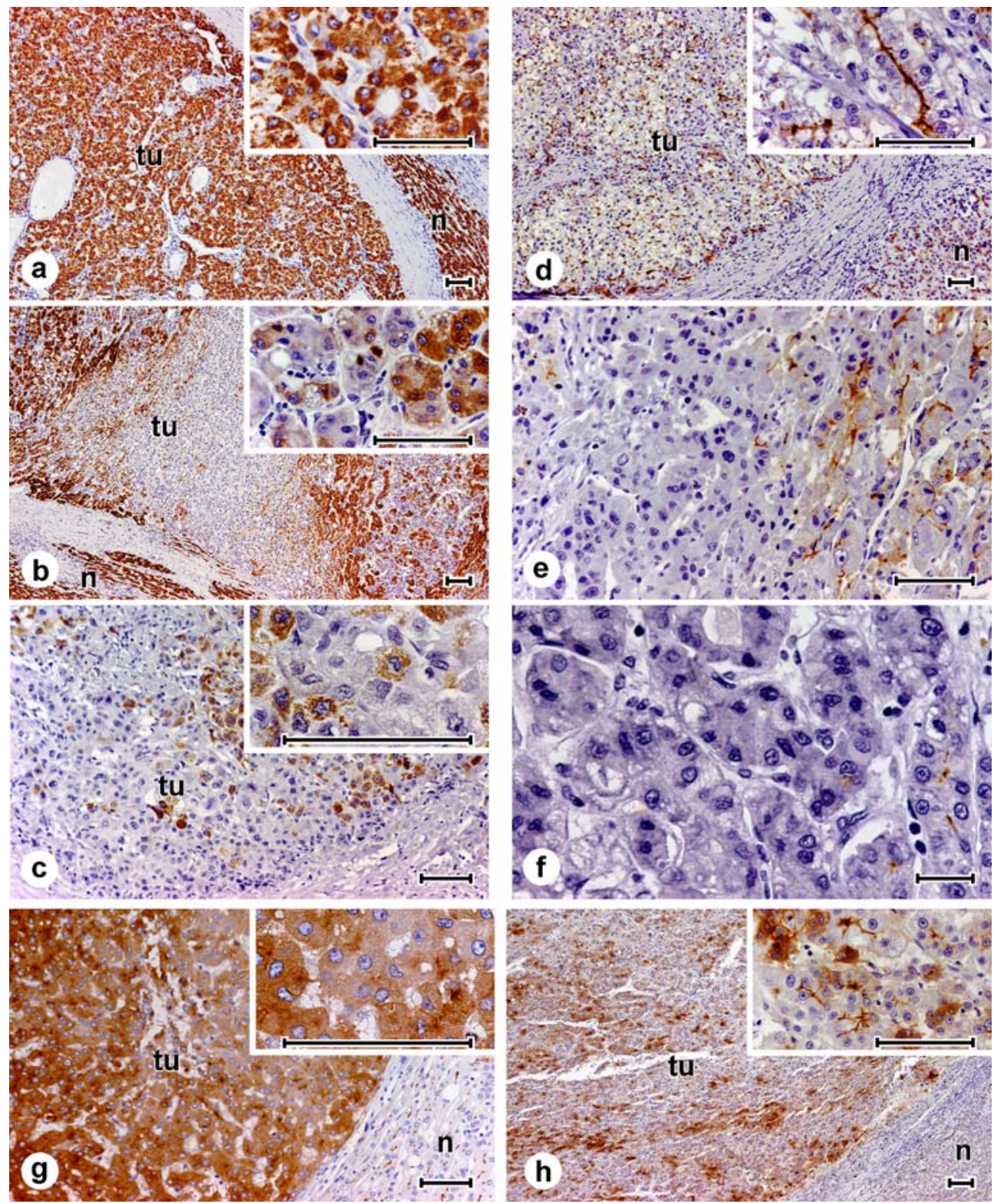

was extensive (score 3 ) in $5(5 \%) \mathrm{HCC}$, partial (score 2) in $23(24 \%) \mathrm{HCC}$, focal (score 1) in $18(19 \%) \mathrm{HCC}$, and absent in $51(52 \%)$ HCC.

Correlations between immunohistochemical features and histopathologic parameters are reported in detail in Table 1. High levels of Hep Par 1 expression (score $=3$ ) were significantly correlated with small tumor size $(p=0.007)$, absence of MVI ( $p=0.03)$, and low HPI $(p=0.04)$. In addition, Hep Par 1 expression decreased with increasing tumor grade in a significant manner ( $p=0.01$; Fig. $2 a)$. All other parameters, including International Union Against Cancer tumor stage and tumor pattern, lacked any significant correlation with Hep Par 1 score. Extensive CD10can labeling (score $\geq 2)$ was more frequent in small HCC $(p=0.04)$ and male patients $(p=0.01)$. Like Hep Par 1, CD10can expression was reduced in poorly differentiated tumors $(p=0.03$; Fig. 2b). CD10cyt staining was not correlated with any clinicopathological feature. A high CIS $(\geq 4)$ showed a highly significant correlation with reduced tumor size $(p=0.05)$, and it was also more frequently observed in male patients $(p=0.04)$. Furthermore, CIS decreased with decreasing tumor differentiation ( $p=0.05$; Fig. $2 c)$.

To assess reproducibility, a subset of immunolabelings was reviewed by another pathologist without particular training. The interobserver agreement was excellent for Hep Par 1 and CD10can labeling $(\kappa=0.87)$ and moderate for CD10cyt labeling $(\kappa=0.53)$.

Correlation of Hep Par 1 and CD10 expression with survival by univariate analysis

To determine the prognostic value of Hep Par 1 and CD10 labeling, the immunohistochemical scores were analyzed as categorical variables using cutoff points that were chosen in a manner to split the study population in two 
Table 1 Relationship between expression of Hep Par 1 or CD10 and pathological factors

\begin{tabular}{|c|c|c|c|c|c|c|c|c|c|}
\hline \multirow[b]{2}{*}{ Factor } & \multirow[b]{2}{*}{$\begin{array}{l}\text { Total number } \\
\text { of patients }\end{array}$} & \multicolumn{8}{|c|}{ Number of patients with } \\
\hline & & $\begin{array}{l}\text { Hep Par } 1 \\
\text { score }=3\end{array}$ & $p$ values $^{\mathrm{a}}$ & $\begin{array}{l}\text { CD10 canalicular } \\
\text { score } \geq 2\end{array}$ & $p$ values & $\begin{array}{l}\text { CD10 cytoplasmic } \\
\text { score } \geq 1\end{array}$ & $p$ values & $\mathrm{CIS}^{\mathrm{b}} \geq 4$ & $p$ values \\
\hline \multicolumn{10}{|c|}{ Tumor size $(\mathrm{cm})$} \\
\hline$\leq 5$ & 58 & $39(67 \%)^{\mathrm{c}}$ & 0.007 & $40(70 \%)$ & 0.04 & $20(51 \%)$ & NS & $29(50 \%)$ & 0.005 \\
\hline$>5$ & 39 & $15(39 \%)$ & & $18(46 \%)$ & & $26(45 \%)$ & & $8(21 \%)$ & \\
\hline \multicolumn{10}{|l|}{ MVI } \\
\hline Absent & 62 & $40(65 \%)$ & 0.03 & $37(60 \%)$ & NS & $29(47 \%)$ & NS & $26(42 \%)$ & NS \\
\hline Present & 35 & $14(40 \%)$ & & $21(60 \%)$ & & $17(49 \%)$ & & $11(31 \%)$ & \\
\hline \multicolumn{10}{|l|}{$\mathrm{HPI}^{\mathrm{d}}$} \\
\hline Low & 46 & $31(67 \%)$ & 0.04 & $30(65 \%)$ & NS & $23(50 \%)$ & NS & $21(46 \%)$ & NS \\
\hline High & 51 & $23(45 \%)$ & & $28(55 \%)$ & & $23(45 \%)$ & & $16(31 \%)$ & \\
\hline
\end{tabular}

NS Statistically not significant

${ }^{\text {a }}$ Two-sided $p$ values obtained by Fisher's exact test, which are considered to be significant if $p<0.05$, of marginal significance if $0.05 \leq p<0.1$, and not significant if $p \geq 0.1$

${ }^{\mathrm{b}}$ CIS (score Hep Par 1 staining+score CD10 canalicular staining-score CD10 cytoplasmic staining)

${ }^{\mathrm{c}}$ Percentages are computed with regard to number of patients for a given factor category

${ }^{\mathrm{d}}$ HPI according to Lauwers et al. [23]

groups of similar size. To detect a possible bias resulting from the type of surgery, univariate and multivariate survival analyses were done not only on the whole study population but also on the subgroup of patients with partial liver resection. On univariate analysis (Table 2) of the whole cohort, Hep Par 1 expression and CD10can labeling were significantly associated with overall survival $(p=0.0005$ and 0.02 , respectively). Indeed, median survival time of patients with tumors showing a strong Hep Par 1 staining (score $=3$ ) was more than 100 months in contrast to patients with a score of $<3$ who survived only 15 months (Fig. 3a). Similarly, survival of patients with an at least moderate CD10can staining (score $\geq 2$ ) was 54 months in contrast to 17 months for the other patients (Fig. 3b). Similar results were found in the subgroup of patients with partial liver resection, who showed also a significant association of strong Hep Par 1 staining with longer median overall survival ( 33 vs. 14 months, $p=0.03$ ). There was a trend toward better overall survival among patients with a CD10can score of $\geq 2$ ( 28 vs. 16 months, $p=0.09$ ). In contrast,
Fig. 2 Correlation between Edmondson and Steiner's tumor grade and immunohistochemical scores: Hep Par 1 expression is significantly decreased in grades 3 and 4 carcinomas (a). CD10can expression is highly decreased in grade 4 tumors (b). Combined immunohistochemical score (CIS=Hep Par 1 score + CD10can score - CD10cyt score) shows a progressive decrease with increasing tumor grade (c). $p$ values were determined using Kruskal-Wallis tests
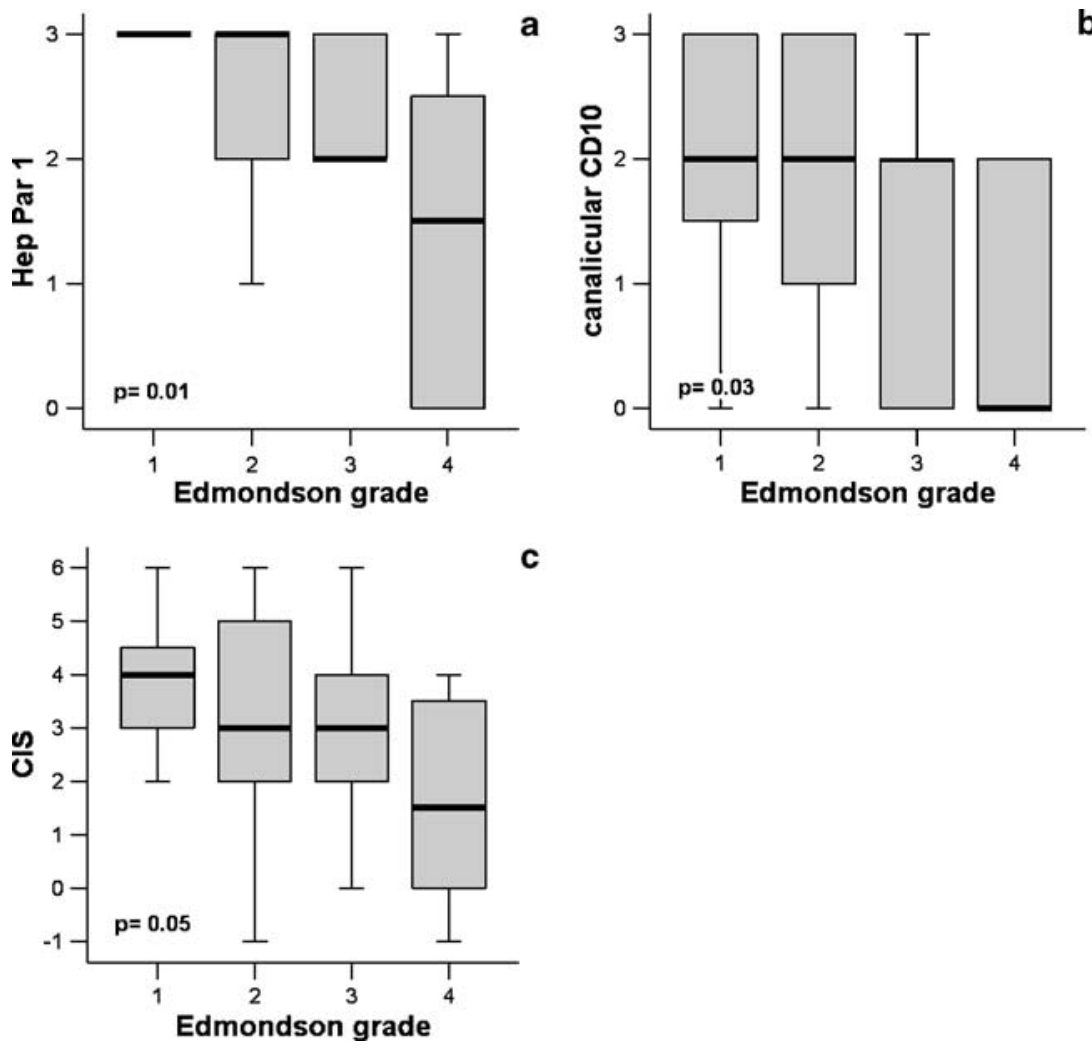
Table 2 Univariate analysis of the immunohistochemical factors

\begin{tabular}{|c|c|c|c|c|c|c|c|c|}
\hline \multirow[b]{2}{*}{$\begin{array}{l}\text { Immunohistochemical } \\
\text { score }\end{array}$} & \multicolumn{4}{|l|}{ All patients } & \multicolumn{4}{|c|}{ Only patients treated by partial resection } \\
\hline & $\begin{array}{l}\text { Number of } \\
\text { patients (censored) }\end{array}$ & $\begin{array}{l}\text { Median survival } \\
\text { time (month) }\end{array}$ & $\begin{array}{l}95 \% \mathrm{CI} \\
\text { (month) }\end{array}$ & $p$ values $^{\mathrm{a}}$ & $\begin{array}{l}\text { Number of } \\
\text { patients (censored) }\end{array}$ & $\begin{array}{l}\text { Median survival } \\
\text { time (month) }\end{array}$ & $\begin{array}{l}95 \% \mathrm{CI} \\
\text { (month) }\end{array}$ & $p$ values \\
\hline \multicolumn{9}{|l|}{ Hep Par 1} \\
\hline$<3$ & $43(9)$ & 15 & $9-21$ & 0.0005 & $36(6)$ & 14 & $6-22$ & 0.03 \\
\hline$=3$ & $54(32)$ & $>100$ & - & & $36(18)$ & 33 & $6-60$ & \\
\hline \multicolumn{9}{|c|}{ CD10 canalicular staining } \\
\hline$<2$ & $39(11)$ & 17 & $7-27$ & 0.02 & $31(7)$ & 16 & $6-26$ & 0.09 \\
\hline$\geq 2$ & $58(30)$ & 54 & $24-84$ & & $41(17)$ & 28 & $4-52$ & \\
\hline \multicolumn{9}{|c|}{ CD10 cytoplasmic staining } \\
\hline$<1$ & $51(24)$ & 31 & $17-45$ & NS & $34(13)$ & 27 & $10-44$ & NS \\
\hline$\geq 1$ & $46(17)$ & 27 & $11-43$ & & 38 (11) & 16 & $0-33$ & \\
\hline \multicolumn{9}{|l|}{$\mathrm{CIS}^{\mathrm{b}}$} \\
\hline$<4$ & $60(16)$ & 17 & $9-25$ & 0.0004 & $48(9)$ & 16 & $13-19$ & 0.004 \\
\hline$\geq 4$ & $37(25)$ & $>80$ & - & & $24(15)$ & $>80$ & & \\
\hline
\end{tabular}

NS Statistically not significant

${ }^{a}$ Two-sided $p$ values obtained by log-rank test, which are considered to be significant if $p<0.05$, of marginal significance if $0.05 \leq p<0.1$, and not significant if $p \geq 0.1$

${ }^{\mathrm{b}}$ CIS (score Hep Par 1 staining + score CD10 canalicular staining - score CD10 cytoplasmic staining)

there was only a weak association between CD10cyt labeling and overall survival in the whole cohort $(p=0.2)$ and in the subgroup of patients with partial resection $(p=0.3)$. Patients with CD10cyt positive tumors tended to have a reduced overall survival as compared to patients with CD10cyt negative tumors.
Fig. 3 Overall survival curves of all 97 patients who underwent either partial hepatectomy with curative intent or total hepatectomy with liver transplantation according to Hep Par 1 score (a), CD10can score (b), combined immunohistochemical score $(\mathrm{CIS}=$ Hep Par 1 score + CD10can score - CD10cyt score) (c). Overall survival curves of the subgroup of 72 patients who underwent partial hepatectomy with curative intent according to combined immunohistochemical score (d). $p$ values were determined using the log-rank test
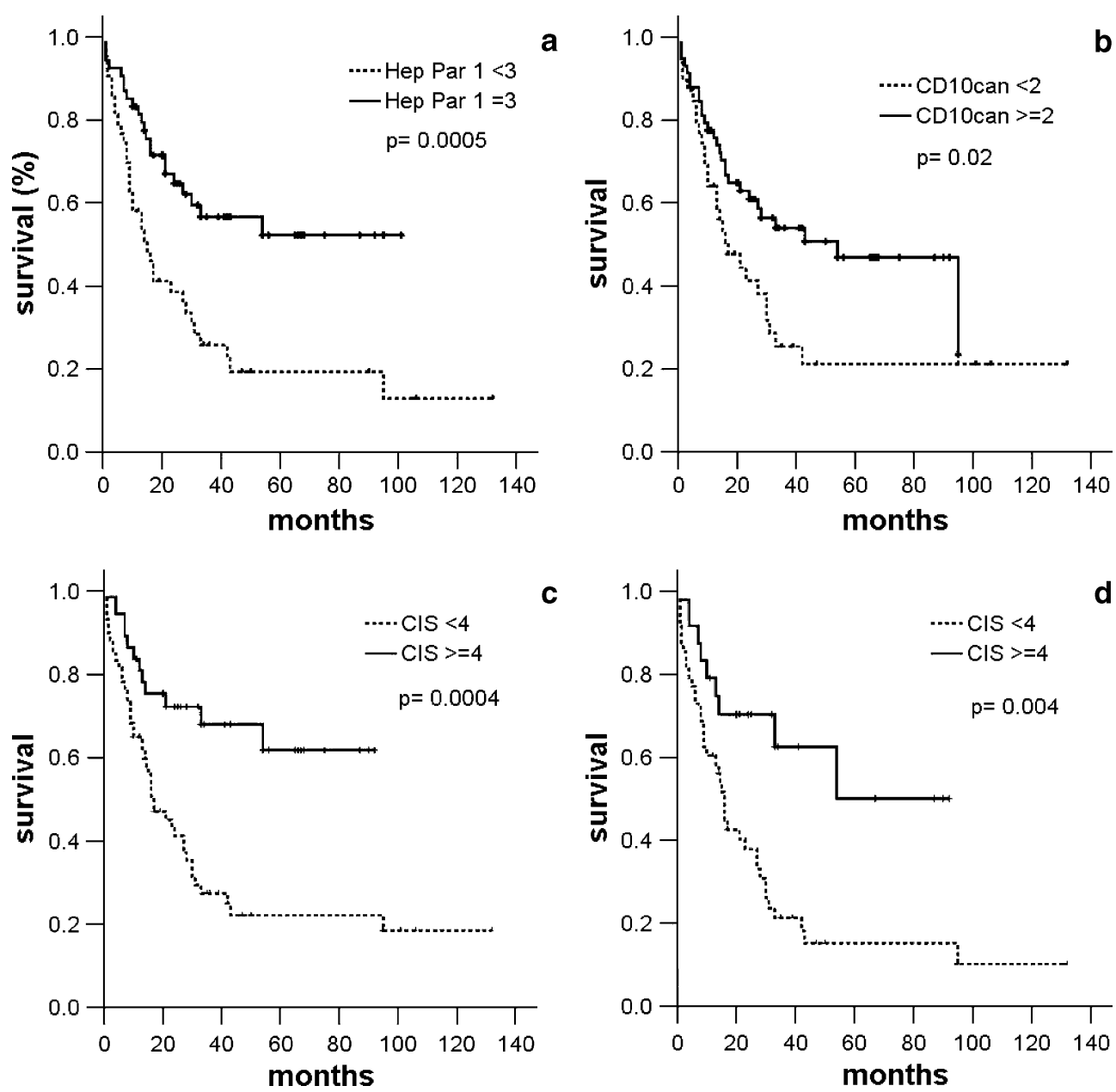
To be able to use these immunohistochemical parameters in a routine clinical setting, they were combined into a simple score according to the following formula: CIS = Hep Par 1 score + CD10can score - CD10cyt score. On univariate analysis, there was a highly significant correlation between CIS and overall survival in both the whole cohort $(p=0.0004)$ and the patients with partial liver resection $(p=0.01)$. In the whole study population, patients with a CIS $\geq 4$ survived more than 80 months whereas the median overall survival of the others was only 17 months (Fig. 3c). Similar median overall survival times were found in the subgroup of partially resected patients ( 80 vs. 16 months; Fig. 3d).

Multivariate survival analysis

Multivariate analysis was performed using several Cox regression models with a limited number of covariates, because the number of outcome events did not allow the evaluation of all pertinent parameters at once. Prognostic variables which had been at least marginally significant (log-rank test $<0.1$ ) in univariate analysis (tumor size, stage, multifocality, and grade as well as MVI, HPI, and surgical margins; Table 3) were further analyzed in various Cox regression models using backward selection with $p<0.05$ as the exit criterion. Following clinicopathological parameters had no impact on survival in univariate analysis and were not further assessed in multivariate models: preoperative transaminase levels and cholestatic parameters, viral hepatitis B and $\mathrm{C}$, alcohol-related liver disease, cigarette smoking, sex, age, and tumor growth pattern. Although not statistically significant on univariate analysis in our series, concomitant liver cirrhosis and preoperative AFP levels were included in a multivariate model because they are well-known prognostic markers.

In the models including only clinicopathological parameters, the retained prognostic factors were tumor multifocality and size in the whole cohort and tumor multifocality and grade in the subgroup of patients with liver resection (model 1 from Table 4).

In the model including Hep Par 1, CD10can, and CD10cyt, all three immunohistochemical parameters were retained, and the regression coefficients (whole cohort) were $0.72,0.77$, and 0.78 for Hep Par 1, CD10can, and CD10cyt, respectively (model 2 from Table 4). Taken

Table 3 Univariate analysis of the clinicopathological factors

\begin{tabular}{|c|c|c|c|c|c|c|c|c|}
\hline \multirow[b]{2}{*}{ Factor $^{\mathrm{a}}$} & \multicolumn{4}{|l|}{ All patients } & \multicolumn{4}{|c|}{ Only patients treated by partial resection } \\
\hline & $\begin{array}{l}\text { Number of } \\
\text { patients (censored) }\end{array}$ & $\begin{array}{l}\text { Median survival } \\
\text { time (month) }\end{array}$ & $\begin{array}{l}95 \% \mathrm{CI} \\
\text { (month) }\end{array}$ & $p$ values $^{\mathrm{b}}$ & $\begin{array}{l}\text { Number of } \\
\text { patients (censored) }\end{array}$ & $\begin{array}{l}\text { Median survival } \\
\text { time (month) }\end{array}$ & $\begin{array}{l}95 \% \mathrm{CI} \\
\text { (month) }\end{array}$ & $p$ values \\
\hline \multicolumn{9}{|c|}{ Tumor size } \\
\hline$\leq 5 \mathrm{~cm}$ & $58(33)$ & 54 & $6-102$ & \multirow[t]{2}{*}{0.0006} & $37(17)$ & 33 & $17-49$ & \multirow[t]{2}{*}{0.07} \\
\hline$>5 \mathrm{~cm}$ & $39(8)$ & 14 & $9-19$ & & $35(7)$ & 14 & $8-20$ & \\
\hline III & $29(11)$ & 27 & $14-40$ & \multirow{2}{*}{0.001} & $23(7)$ & 21 & $4-38$ & \multirow{2}{*}{0.06} \\
\hline IV & $35(9)$ & 14 & $9-19$ & & $27(5)$ & 13 & $6-20$ & \\
\hline \multicolumn{9}{|c|}{ Tumor multifocality } \\
\hline solitary & $43(25)$ & 95 & $5-185$ & \multirow[t]{2}{*}{0.001} & $31(15)$ & 33 & $21-45$ & \multirow[t]{2}{*}{0.007} \\
\hline multiple & $54(16)$ & 14 & $9-19$ & & $41(9)$ & 13 & $7-19$ & \\
\hline \multicolumn{9}{|l|}{ MVI } \\
\hline Absent & $62(31)$ & 42 & $27-57$ & \multirow[t]{2}{*}{0.04} & $46(18)$ & 30 & $22-38$ & \multirow[t]{2}{*}{0.2} \\
\hline Present & $35(10)$ & 15 & $8-21$ & & $26(6)$ & 10 & $5-15$ & \\
\hline \multicolumn{9}{|l|}{$\mathrm{HPI}^{\mathrm{c}}$} \\
\hline Low & $46(25)$ & 54 & 8-100 & \multirow[t]{2}{*}{0.03} & $34(15)$ & 42 & $18-66$ & \multirow[t]{2}{*}{0.1} \\
\hline High & $51(16)$ & 16 & $13-19$ & & $38(9)$ & 15 & $11-18$ & \\
\hline \multicolumn{9}{|c|}{ Surgical margins } \\
\hline Negative & $78(37)$ & 33 & $17-49$ & \multirow[t]{2}{*}{0.05} & $56(22)$ & 30 & $18-42$ & \multirow[t]{2}{*}{0.01} \\
\hline Positive & $14(4)$ & 9 & $7-11$ & & $11(2)$ & 9 & $6-12$ & \\
\hline
\end{tabular}

${ }^{\mathrm{a}}$ Following factors did not show a significant impact on survival $(p>0.2)$ and are omitted from this table for simplicity: concomitant liver cirrhosis, etiology of underlying liver pathology (alcohol, hepatitis B, and hepatitis C), as well as preoperative AFP levels, cytolytic, and cholestatic parameters

${ }^{\mathrm{b}}$ Two-sided $p$ values obtained by log-rank test, which are considered to be significant if $p<0.05$, of marginal significance if $0.05 \leq p<0.1$ and NS if $p \geq 0.1$

${ }^{c}$ HPI was computed according to Lauwers et al. [23] 
together with the selected clinicopathological parameters, Hep Par 1 and CD10can were retained in the models (models 3 and 4 from Table 4, respectively). However, $p$ values and relative risk were lower for Hep Par 1 and CD10can alone as compared to CIS (see below).

All models testing CIS in conjunction with different clinicopathological parameters resulted in the retention of the same final variables. Models 5 and 6 from Table 4 are representative examples. CIS was the most significant prognostic factor in both the complete cohort $(p=0.001)$ and the subgroup of liver-resected patients $(p=0,004)$. Patients with a CIS $<4$, as compared to those with a CIS $\geq 4$, had a 3.5fold $(95 \%$ CI $1.7-7.3)$ and a 3.7 -fold (95\% CI 1.5-8.9) increased risk of death in the whole cohort and the subgroup of patients with liver resection, respectively. In the model of the whole cohort, tumor multifocality was the only additional prognostic factor $(p=0.005)$ : patients with multifocal HCC had a 2.4 -fold (95\% CI 1.3 to 4.3 ) increased risk of death as compared to those with solitary tumors. In the model of patients with liver resection only, tumor multifocality $(p=0.02)$ and Edmondson and Steiner's grade $(p=0.04)$ were retained in addition to CIS.

\section{Discussion}

Hepatocellular carcinoma is one of the most common malignant tumors worldwide that has typically poor prognosis regardless of the treatment, and its incidence and mortality are on the rise in Western nations [14]. Clinicians need prognostic indicators to inform their patients and to stratify them for eventual additional therapy. The present study of Hep Par 1 and CD10 expression in HCC by immunohistochemistry was undertaken to identify new prognostic markers for patients treated with curative intent by partial or total hepatectomy, the latter with liver transplantation.

In our study population, strong cytoplasmic Hep Par 1 and CD10can expression, as well as absent CD10cyt labeling, were correlated with longer overall survival. To obtain a practical algorithm for clinical use, these three immunohistochemical parameters were combined into a single immunohistochemical score, which showed a highly significant correlation with overall survival. Multivariate analysis proved CIS to be the most important independent prognostic factor, followed by tumor multifocality and Edmondson and Steiner's grade. Furthermore, CIS is superior to Hep Par 1 or CD10 scores alone with regard to risk assessment. To our knowledge, this is the first study to demonstrate the prognostic value of these markers, although their use is well documented in establishing the diagnosis of HCC [5, 7, 8, 38]. CIS was also the most significant prognostic indicator in the subgroup of patients treated by partial hepatectomy, which excludes the possibility of a bias due to the type of surgery. Unfortunately, the limited number of patients treated by total hepatectomy and the limited duration of follow-up precluded analysis of this subgroup.
A future study is planned to examine in detail the prognostic value of Hep Par 1 and CD10 in this group of patients.

Prognostic indices are usually derived from the final Cox regression model by using the regression coefficients as weights for the corresponding scores [6]. However, we preferred a more simple prognostic index based on a weight of +1 for Hep Par 1 and CD10can and a weight of -1 for CD10cyt scores for two reasons. First, its determination is simple enough to be applicable in a routine setting. Second, as shown by the Cox model, including these three parameters, the absolute value of their regression coefficients is roughly the same (about 0.7 ), which justifies a posteriori such a score. Interestingly, several parameters, such as TNM stage, tumor size, MVI, HPI, and surgical resection margins, which are known in the literature to be of prognostic significance, were not retained in our Cox regression models. This can be explained by correlation of the immunohistochemical variables with these clinicopathological parameters, as shown in Table 1 for tumor size and grade, MVI and HPI. Second, tumor multifocality, which remains significant in our multivariate models, contains partly the same prognostic information as TNM stage, which is based on the former.

The precise biological mechanisms underlying the impact of Hep Par 1 and CD10 expression on prognosis have yet to be identified. It might be linked to the fact that Hep Par 1 and CD10can expressions are indicators of a high degree of differentiation. Indeed, in our series, there was a correlation between the expression of these markers and histological grade. These findings are in line with several reports in the literature $[5,8,21,39]$. Another argument in favor of this hypothesis is the fact that poorly differentiated HCC are known to be devoid of bile canaliculi $[5,7]$. Therefore, these tumors are frequently unreactive with antibodies directed against CD10 which is a neutral endopeptidase located at the luminal aspects of bile canaliculi. To explain the intriguing feature of CD10cyt labeling in the less differentiated and more aggressive tumors, one might also speculate that redistribution of CD10 from the cell surface to the cytoplasm is an indirect sign of lost cell polarity, which is one of the hallmarks of cancer [35]. This redistribution might be the consequence of aberrant glycosylation, alternative splicing, or a truncating mutation of the CD10 gene [33, 36].

In conclusion, our study identifies Hep Par 1 and CD10 expression as the most informative predictors of overall survival in HCC patients treated by partial liver resection with curative intent. Introduction of these parameters in clinical decision making, however, requires confirmation of these preliminary findings in another population of HCC patients. Whether these findings apply also to patients treated with total hepatectomy and liver transplantation as suggested by the present work will require further studies. Future prospective studies are also necessary to determine whether or not the CIS can be helpful for the choice of additional treatment and prediction of prognosis in patients who are beyond curative surgery. 
Acknowledgements We thank M. Gillet (Department of Surgery, CHUV, Lausanne) for the patient data, S. Pampallona (Evolène, Switzerland) for advice on statistical analysis, and P. Chaubert and P. Shaw (Department of Pathology, CHUV, Lausanne) for help in immunohistochemistry and for critical review of the manuscript, respectively.

This study has been conducted in accordance with current Swiss law and been approved by the ethical committee of our institution.

\section{References}

1. Adachi E, Maeda T, Kajiyama K, Kinukawa N, Matsumata T, Sugimachi K, Tsuneyoshi M (1996) Factors correlated with portal venous invasion by hepatocellular carcinoma: univariate and multivariate analyses of 232 resected cases without preoperative treatments. Cancer 77:2022-2031

2. Anthony PP (2001) Hepatocellular carcinoma: an overview. Histopathology 39:109-118

3. Armitage P, Berry G, Matthews JNS (1987) Statistical methods in medical research, 2nd edn. Blackwell, Oxford

4. Bene MC, Faure GC (1997) CD10 in acute leukemias. GEIL (Groupe d'Etude Immunologique des Leucemies). Haematologica 82:205-210

5. Borscheri N, Roessner A, Rocken C (2001) Canalicular immunostaining of neprilysin (CD10) as a diagnostic marker for hepatocellular carcinomas. Am J Surg Pathol 25:1297-1303

6. Christensen E (1987) Multivariate survival analysis using Cox's regression model. Hepatology 7:1346-1358

7. Chu P, Arber DA (2000) Paraffin-section detection of CD10 in 505 nonhematopoietic neoplasms. Frequent expression in renal cell carcinoma and endometrial stromal sarcoma. Am J Clin Pathol 113:374-382

8. Chu PG, Ishizawa S, Wu E, Weiss LM (2002) Hepatocyte antigen as a marker of hepatocellular carcinoma: an immunohistochemical comparison to carcinoembryonic antigen, CD10, and alpha-fetoprotein. Am J Surg Pathol 26:978-988

9. Colombo M (1992) Hepatocellular carcinoma. J Hepatol $15: 225-236$

10. Cox D (1972) Regession models and life tables. J R Stat Soc B $34: 187-220$

11. Dragovic T, Deddish PA, Tan F, Weber G, Erdos EG (1994) Increased expression of neprilysin (neutral endopeptidase 24.11) in rat and human hepatocellular carcinomas. Lab Invest 70 : $107-113$

12. Dragovic T, Sekosan M, Becker RP, Erdos EG (1997) Detection of neutral endopeptidase 24.11 (neprilysin) in human hepatocellular carcinomas by immunocytochemistry. Anticancer Res 17: 3233-3238

13. Edmondson H, Steiner P (1954) Primary carcinoma of the liver: a study of 100 cases among 48,900 necropsies. Cancer 7:462-503

14. El-Serag HB, Mason AC (1999) Rising incidence of hepatocellular carcinoma in the United States. N Engl J Med 340:745750

15. Greenwood M (1926) Reports on public health and medical subjects: the natural duration of cancer. HMSO 33:1-16

16. Hamilton SR, Aaltonen LA (eds) (2000) Pathology and genetics of tumors of the digestive system. IARC Press, Lyon

17. Hanazaki K, Kajikawa S, Koide N, Adachi W, Amano J (2001) Prognostic factors after hepatic resection for hepatocellular carcinoma with hepatitis $\mathrm{C}$ viral infection: univariate and multivariate analysis. Am J Gastroenterol 96:1243-1250
18. Inagawa $\mathrm{S}$, Itabashi $\mathrm{M}$, Adachi $\mathrm{S}$, Kawamoto $\mathrm{T}$, Hori $\mathrm{M}$, Shimazaki J, Yoshimi F, Fukao K (2002) Expression and prognostic roles of beta-catenin in hepatocellular carcinoma: correlation with tumor progression and postoperative survival. Clin Cancer Res 8:450-456

19. Kaplan EL, Meier P (1958) Nonparametric estimation from incomplete observations. J Am Stat Assoc 53:457-481

20. Kobayashi T, Sugawara Y, Shi YZ, Makuuchi M (2002) Telomerase expression and p53 status in hepatocellular carcinoma. Am J Gastroenterol 97:3166-3171

21. Kumagai I, Masuda T, Sato S, Ishikawa K (2001) Immunoreactivity to monoclonal antibody, Hep Par 1, in human hepatocellular carcinomas according to histopathological grade and histological pattern. Hepatol Res 20:312-319

22. Lamps LW, Folpe AL (2003) The diagnostic value of hepatocyte paraffin antibody 1 in differentiating hepatocellular neoplasms from nonhepatic tumors: a review. Adv Anat Pathol 10:39-43

23. Lauwers GY, Terris B, Balis UJ, Batts KP, Regimbeau JM, Chang Y, Graeme-Cook F, Yamabe H, Ikai I, Cleary KR, Fujita S, Flejou JF, Zukerberg LR, Nagorney DM, Belghiti J, Yamaoka Y, Vauthey JN (2002) Prognostic histologic indicators of curatively resected hepatocellular carcinomas: a multi-institutional analysis of 425 patients with definition of a histologic prognostic index. Am J Surg Pathol 26:25-34

24. Lauwers GY, Vauthey JN (1998) Pathological aspects of hepatocellular carcinoma: a critical review of prognostic factors. Hepatogastroenterology 45:1197-1202

25. Leong AS, Sormunen RT, Tsui WM, Liew CT (1998) Hep Par 1 and selected antibodies in the immunohistological distinction of hepatocellular carcinoma from cholangiocarcinoma, combined tumours and metastatic carcinoma. Histopathology 33:318-324

26. Medina-Franco H, Sellers MT, Eckhoff DE, Bynon JS, Urist MM, Heslin MJ (2001) Multimodality treatment for patients with hepatocellular carcinoma: analysis of prognostic factors in a single Western institution series. J Gastrointest Surg 5:638645

27. Minervini MI, Demetris AJ, Lee RG, Carr BI, Madariaga J, Nalesnik MA (1997) Utilization of hepatocyte-specific antibody in the immunocytochemical evaluation of liver tumors. Mod Pathol 10:686-692

28. Morrison C, Marsh W Jr, Frankel WL (2002) A comparison of CD10 to pCEA, MOC-31, and hepatocyte for the distinction of malignant tumors in the liver. Mod Pathol 15:1279-1287

29. Nzeako UC, Goodman ZD, Ishak KG (1996) Hepatocellular carcinoma in cirrhotic and noncirrhotic livers. A clinicohistopathologic study of 804 North American patients. Am J Clin Pathol 105:65-75

30. Okuda K (2002) Natural history of hepatocellular carcinoma including fibrolamellar and hepato-cholangiocarcinoma variants. J Gastroenterol Hepatol 17:401-405

31. Sato Y, Itoh F, Hareyama M, Satoh M, Hinoda Y, Seto M, Ueda R, Imai K (1999) Association of cyclin D1 expression with factors correlated with tumor progression in human hepatocellular carcinoma. J Gastroenterol 34:486-493

32. Schoniger-Hekele M, Muller C, Kutilek M, Oesterreicher C, Ferenci P, Gangl A (2001) Hepatocellular carcinoma in Central Europe: prognostic features and survival. Gut 48:103-109

33. Sezaki N, Ishimaru F, Tabayashi T, Kataoka I, Nakase K, Fujii K, Kozuka T, Nakayama H, Harada M, Tanimoto M (2003) The type $1 \mathrm{CD} 10$ /neutral endopeptidase 24.11 promoter: functional characterization of the 5'-untranslated region. Br J Haematol 123:177-183 
34. Sobin LH, Wittekind C (eds) (2002) TNM classification of malignant tumors. Wiley, New York

35. Thiery JP, Humbert P, Russell S, Richardson H (2002) Epithelial-mesenchymal transitions in tumour progression Dlg, Scribble and Lgl in cell polarity, cell proliferation and cancer. Nat Rev Cancer 2:442-454

36. Turner AJ, Isaac RE, Coates D (2001) The neprilysin (NEP) family of zinc metalloendopeptidases: genomics and function. Bioessays 23:261-269

37. Turner AJ, Tanzawa K (1997) Mammalian membrane metallopeptidases: NEP, ECE, KELL, and PEX. Faseb J 11:355-364
38. Wennerberg AE, Nalesnik MA, Coleman WB (1993) Hepatocyte paraffin 1: a monoclonal antibody that reacts with hepatocytes and can be used for differential diagnosis of hepatic tumors. Am J Pathol 143:1050-1054

39. Wu PC, Fang JW, Lau VK, Lai CL, Lo CK, Lau JY (1996) Classification of hepatocellular carcinoma according to hepatocellular and biliary differentiation markers. Clinical and biological implications. Am J Pathol 149:1167-1175

40. Xiao SY, Wang HL, Hart J, Fleming D, Beard MR (2001) cDNA arrays and immunohistochemistry identification of CD10/CALLA expression in hepatocellular carcinoma. Am J Pathol 159:1415-1421 